\title{
Taste breaking effects in scalar meson correlators
}

\section{Bernard}

Department of Physics, Washington University, St. Louis, MO 63130, USA

E-mail: cb@ lump.wust l . edu

C. DeTar* and Ziwen Fu

Department of Physics, University of Utah, Salt Lake City, UT 84112, USA

E-mail: detar@physics.utah.edu, kevin@physics.utah.edu

\section{S. Prelovsek}

Department of Physics, University of Ljubljana, Jadranska 19, Ljubljana, Slovenia

and J. Stefan Institute, Jamova 39, PO Box 300, 1001, Ljubljana, Slovenia

E-mail: Sasa.Prelovsek@ijs.si

As a consistency check of the staggered-fermion fourth-root approximation, we analyze the $a_{0}$ and $f_{0}$ correlators, including the effects of two-meson taste-multiplet intermediate states. Rooted staggered chiral perturbation theory describes the contributions from the pseudoscalar taste multiplets in terms of only a few low energy constants, which have all been previously determined by the MILC collaboration. In previous work one of us (Prelovsek) showed that the two-meson "bubble" contributions could explain the observed anomalies in the lattice data for the isovector $a_{0}$ channel. In the present work we extend this analysis to the $f_{0}$ channel. On a MILC collaboration lattice ensemble at $0.12 \mathrm{fm}$ with $2+1$ flavors of Asqtad-improved staggered fermions, we have made new measurements of correlators in both channels for a variety of momenta. A fit to these correlators gives low energy constants that are reasonably consistent with previous determinations by the MILC collaboration.

XXIV International Symposium on Lattice Field Theory

July 23-28 2006

Tucson, Arizona, USA

\footnotetext{
* Speaker.
} 


\section{Introduction}

When up and down quark masses are sufficiently light, the isovector $\left(a_{0}\right)$ and isosinglet $\left(f_{0}\right)$ scalar meson correlators are dominated at large distances by two-body states composed of $\pi, K$, and $\eta$. In the lattice staggered fermion formulation, residual fermion doubling results in a sixteenmember taste multiplet for each meson. When this symmetry is broken at nonzero lattice spacing, the multiplet is split, resulting in a proliferation of two-body intermediate states, thereby complicating the analysis of the correlators. To make matters worse, many of these states are lattice artifacts with unphysical masses, and some have ghost (negative) weights. Thus we see nonlocalities in the form of unphysical long-range contributions to the correlators, and we see violations of unitarity in the form of unphysical and negative norm intermediate states. Such artifacts are expected to disappear in the continuum limit.

Fortunately, these complexities are described by staggered chiral perturbation theory ( $\chi \mathrm{PT})$. The rooted version of this theory $(\mathrm{rS} \chi \mathrm{PT})[1,2]$ provides a strict framework in which to analyze these two-body contributions in terms of just a few low energy constants - constants that are also determined in an independent staggered chiral analysis of the pseudoscalar meson masses and decay constants [3].

Thus the study of scalar correlators provides an explicit example of the anticipated diseases of the fourth-root approximation and the manner in which they are cured in the continuum limit. Further, it provides another test of the ability of $\mathrm{rS} \chi \mathrm{PT}$ to model rooted staggered fermion QCD. Previous tests include the aforementioned fits to the pseudoscalar meson masses and decay constants and a comparison of the topological susceptibility measured in rooted staggered fermion $\mathrm{QCD}$ and in $\mathrm{rS} \chi \mathrm{PT}[4]$.

\section{Pseudoscalar meson multiplet}

The $a_{0}$ channel was studied in recent years in staggered fermion QCD by the MILC collaboration [5] and UKQCD collaboration [6]. Both groups found that the correlator appeared to contain states with energies well below possible combinations of known mesons. At Lattice 2005 one of us showed that $\mathrm{rS} \chi \mathrm{PT}$ provides a simple explanation [7,8]. In Fig. 1 we show taste splittings of the low-lying pseudoscalar mesons for the MILC $a=0.125 \mathrm{fm}$ ensemble, as predicted in $\mathrm{rS} \chi \mathrm{PT}$. In the case of the pion the predictions were confirmed in lattice measurements [5, 9]. Of particular concern are the $\eta$ and $\eta^{\prime}$ multiplets. They are mixed by the anomaly. But the anomaly is a taste singlet. So only the taste-singlet members are proper candidates for the physical states. The other taste members are unmixed at tree level. We have arbitrarily labeled the lighter member $\eta$ and heavier member $\eta^{\prime}$. The taste-axial-vector and vector hairpin couplings mix the $\eta$ 's weakly. We ignore that effect in the figure, but take it fully into account in our analysis.

Now, let us examine the various two-meson contributions to the $a_{0}$ correlator. In proper QCD the lowest intermediate state has mass $m_{\pi}+m_{\eta}$. In staggered QCD the physical $a_{0}$ is a taste singlet, so we need to examine the various two-meson contributions to the taste-singlet correlator. Taste symmetry requires that both pseudoscalar mesons in an intermediate state have the same taste assignment. In particular, the $\pi \eta$ intermediate states include a taste-pseudoscalar pion and a 


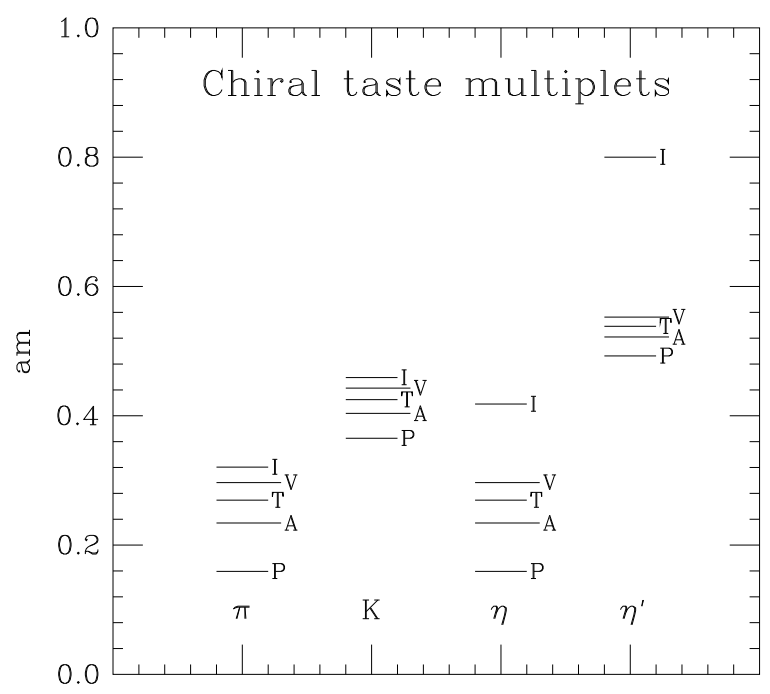

Figure 1: Taste multiplets for $\pi, K, \eta$, and $\eta^{\prime}$ for the study ensemble. The letters indicate the taste symmetry assignments, grouped as predicted by Lee and Sharpe [10].

taste-pseudoscalar eta with a mass equal to twice the Goldstone pion mass. Other taste pairs are similarly light. Thus we expect an anomalous low energy contribution to the correlator.

\section{Threshold weights}

Explicit expressions were presented in $[7,8]$ for the two-meson contribution to the $a_{0}$ correlator, including all tastes. We have extended this analysis to the $f_{0}$ correlator [11]. (See also Sec. VI of Ref. [2] for a description of the result in the one-flavor case.) The expressions (not written here) consist of several two-body threshold contributions with weights depending on the number of flavors and the replica factor [12] needed to correct for the unwanted taste multiplicity. If we ignore mixings induced by the taste axial-vector and vector hairpins, the weights for the physical $2+1$ flavor case are given in Table 1. In the continuum limit in the $a_{0}$ channel, all thresholds but the taste-singlet $\pi \eta$ become degenerate and the axial-vector and vector hairpin mixings vanish. As is evident from the table, the weights add up to zero. The ghost threshold has eaten the unphysical thresholds, and only the physical taste-singlet $\pi \eta$ survives. In the $f_{0}$ channel, all the $\pi \pi$ thresholds become degenerate leaving a proper weight of 3 for the three charge assignments for the physical pions.

For the single-flavor case there is no Goldstone boson, since the one pseudoscalar is lifted by the anomaly. Yet at nonzero lattice spacing there is a multiplet of near Goldstone bosons composed of taste components that are unmixed by the anomaly. In the continuum limit the two-body thresholds for these states become degenerate and the weights add to zero in the $f_{0}$ channel as can be seen from Table 2. Thus the unphysical states decouple from this channel.

\section{Fits and Results}

Measurements were done on a single ensemble of 510 gauge configurations of dimension $24^{3} \times 64$, generated by the MILC collaboration with $2+1$ flavors of staggered quarks of bare mass 


\begin{tabular}{lrr} 
taste & $a_{0}$ & $f_{0}$ \\
\hline$I$ & $2 / 3$ & $1 / 4$ \\
$I 0$ & $-15 / 8$ & -1 \\
$V$ & $4 / 8$ & $4 / 4$ \\
$T$ & $6 / 8$ & $6 / 4$ \\
$A$ & $4 / 8$ & $4 / 4$ \\
$P$ & $1 / 8$ & $1 / 4$
\end{tabular}

Table 1: Two-body threshold weights for $2+1$ flavors in the $a_{0}$ and $f_{0}$ channels. The $a_{0}$ weights apply to the $\pi \eta$ contributions for tastes $I, P, V, A, T$, and the $f_{0}$ weights apply to the $\pi \pi$ contributions. Here the tastesinglet $\eta$ is the physical state. For the $a_{0}$ the weight $I 0$ denotes the contribution from the taste-singlet pion plus the bare taste-singlet $\eta$ (unshifted by the anomaly). For the $f_{0}$ the weight $I 0$ denotes the contribution from two bare taste-singlet etas.

\begin{tabular}{lr} 
taste & $f_{0}$ \\
\hline$I 0$ & $-15 / 8$ \\
$V$ & $4 / 8$ \\
$T$ & $6 / 8$ \\
$A$ & $4 / 8$ \\
$P$ & $1 / 8$
\end{tabular}

Table 2: Two-body threshold weights for the single flavor case $f_{0}$ channel. The notation is the same as Table 1.

$a m_{u d}=0.005$ and $a m_{s}=0.05$ at a lattice spacing of approximately $a=0.125 \mathrm{fm}$. For this ensemble the pi/rho mass ratio is 0.303 .

We measured the correlator of scalar densities $\bar{\psi} \psi$ for the isovector and isoscalar channels and for five low-lying total momenta $p$. The correlators were fit to the expressions

$$
\begin{aligned}
& C_{a 0}(p, t)=f_{\text {meson }, a 0}(p, t)+f_{\text {bubble }, a 0}(p, t) \\
& C_{f 0}(p, t)=f_{\text {meson }, f 0}(p, t)+f_{\text {bubble }, f 0}(p, t)
\end{aligned}
$$

and where the single-meson contribution is modeled by a single pole of each parity,

$$
\begin{aligned}
& f_{\text {meson }, a 0}(p, t)=b_{a 0}(p) \exp \left[-E_{a 0}(p) t\right]+b_{\pi, A}(p)(-)^{t} \exp \left[-E_{\pi, A}(p) t\right]+\left(N_{t}-t\right) \\
& \left.f_{\text {meson }, f 0}(p, t)=c_{0} \delta_{(} p, 0\right)+b_{f 0}(p) \exp \left[-E_{f 0}(p) t\right]+b_{\eta, A}(p)(-)^{t} \exp \left[-E_{\eta, A}(p) t\right]+\left(N_{t}-t\right),
\end{aligned}
$$

and where the "bubble" or two-meson contributions are dictated by $\operatorname{rS} \chi \mathrm{PT}[8,7,11]$. The meson contributions require 13 parameters (four for the masses, two each for the momentum dependence of the four coefficients, and one for the zero-momentum vacuum disconnected term in the $f_{0}$ ). For each meson energy we assume a standard dispersion relation $E(p)^{2}=m^{2}+p^{2}$. The meson masses in the two-meson bubble contributions are fixed from other spectroscopic studies plus the known taste splittings [5, 3]. There remain three low energy constants from $\mathrm{rS} \chi \mathrm{PT}$, namely,

$$
\mu=m_{\pi}^{2} /\left(2 m_{u, d}\right), \quad \delta_{A}=a^{4} \delta_{A}^{\prime}, \delta_{V}=a^{4} \delta_{V}^{\prime}
$$


All three parameters were previously obtained from a study of meson masses and decays [3]. We found it necessary to fix (through a Bayesian prior) the vector hairpin constant $\delta_{V}$ to the value obtained in that study. The remaining two constants were then adjusted with only loose priors.

The resulting best fits are shown in Figs. 2, 3, and 4.

\begin{tabular}{lrr} 
quantity & our result & meson mass, decay \\
\hline$r_{1} m_{\pi}^{2} /\left(2 m_{u, d}\right)$ & $8.2(1.1)$ & 6.7 \\
$\delta_{V}$ & $($ prior $)$ & $-0.016(23)$ \\
$\delta_{A}$ & $-0.056(7)$ & $-0.040(6)$
\end{tabular}

Table 3: Comparison of our fit parameters for the $\mathrm{rS} \chi \mathrm{PT}$ low energy constants with results from [3]

The agreement is worse if we used the value of $r_{1} \mu$ in the three-flavor chiral limit $(\approx 4.5)$ from those fits, rather than the higher-order $m_{\pi}^{2} /\left(2 m_{u d}\right)$, suggesting, perhaps, that a higher order calculation of the bubble contribution might improve the agreement.

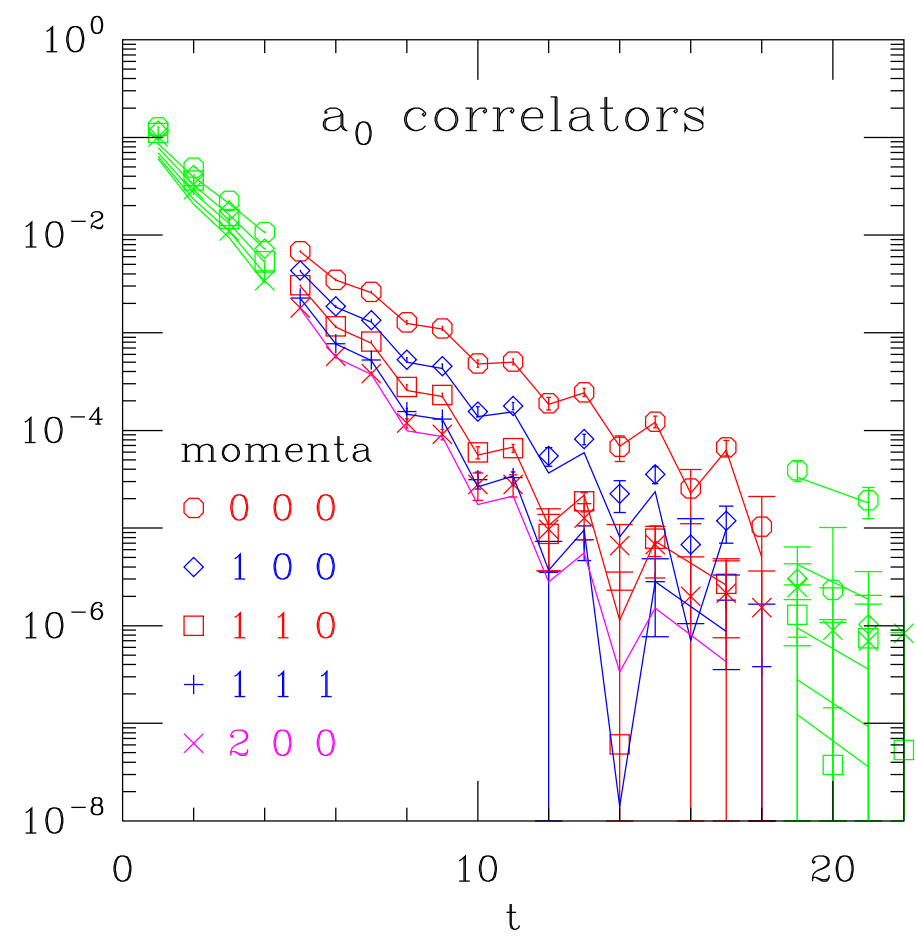

Figure 2: Fit to the $a_{0}$ correlator for five momenta.

\section{Conclusion}

We have found that rooted staggered chiral perturbation theory together with a couple of explicit low-lying mesons provides a reasonably consistent accounting of the $a_{0}$ and $f_{0}$ correlators for several low momenta. The spurious long-range spectral components are lattice artifacts, which the theory predicts will disappear in the continuum limit. 


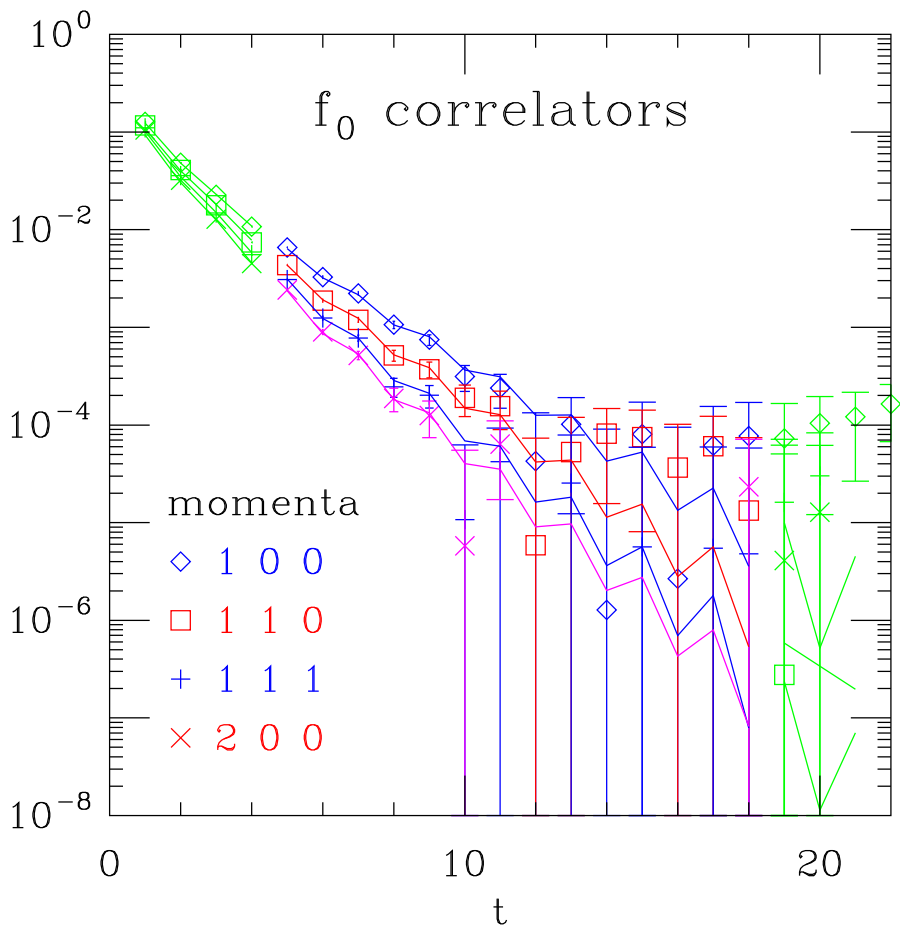

Figure 3: Fit to the $f_{0}$ correlator for four momenta.

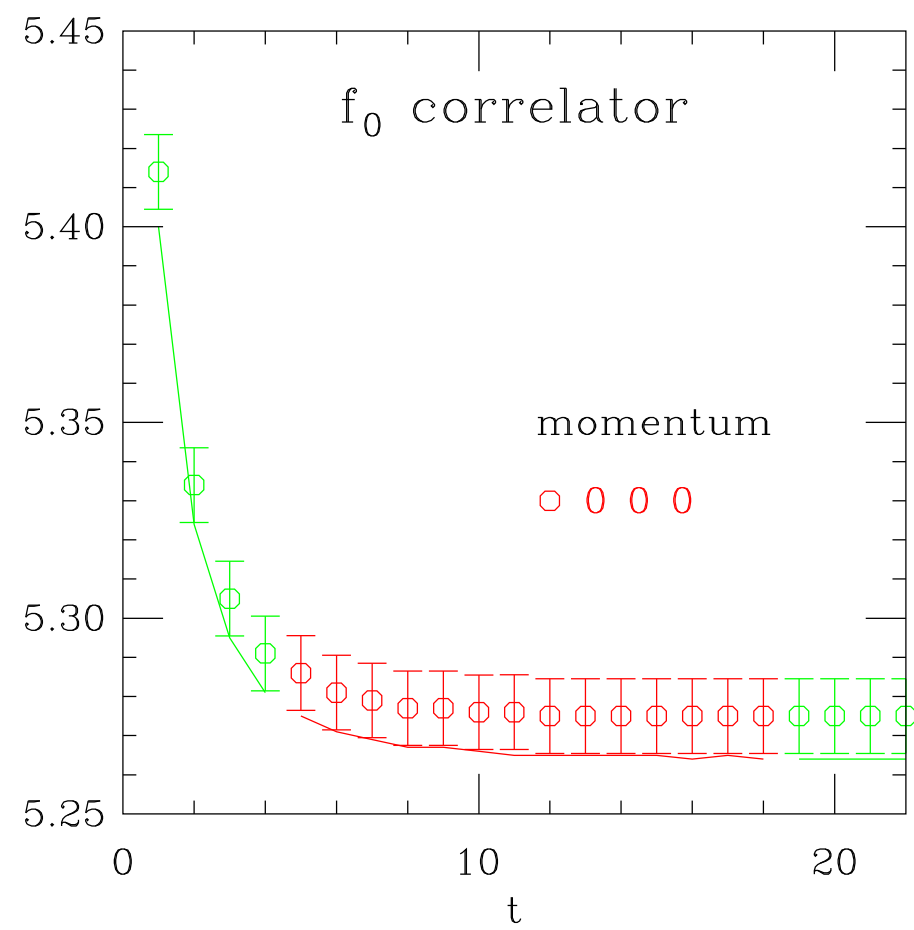

Figure 4: Fit to the $f_{0}$ correlator for zero momentum. 
Computing support from the Utah Center for High Performance Computing is gratefully acknowledged. This work was supported by the US NSF and DOE. We thank the MILC collaboration for the use of its lattice ensembles.

\section{References}

[1] C. Aubin and C. Bernard, Phys. Rev. D 68, 034014 (2003), [hep-lat/0304014]; C. Aubin and C. Bernard, ibid., 074011 (2003), [hep-lat/0306026];

[2] C. Bernard, Phys. Rev. D 73, 114503 (2006) [hep-lat/0603011].

[3] C. Aubin et al. (MILC Collaboration), Phys. Rev. D 70, 114501 (2004), [hep-lat/0407028].

[4] B. Billeter, C. DeTar, and J. Osborn, Phys. Rev. D 70, 077502 (2004), [hep-lat/0406032].

[5] C. Aubin et al. (MILC Collaboration), Phys. Rev. D 70, 094505 (2004), [hep-lat/0406032].

[6] E.B. Gregory, A.C. Irving, C.C. McNeile, S. Miller, and Z.Sroczynski, PoS (LAT2005) 027 (2006), [hep-lat/0510066].

[7] S. Prelovsek, PoS (LAT2 005 ) 085 (2006), [hep-lat/0509083].

[8] S. Prelovsek, Phys. Rev. D 73, 014506 (2006), [hep-lat/0510080].

[9] C.W. Bernard et al. (MILC Collaboration), Phys. Rev. D 64, 054506 (2001), [hep-lat/0104002].

[10] W.-J. Lee and S.R. Sharpe, Phys. Rev. D 60, 114503 (1999), [hep-lat/9905023].

[11] C. Bernard, C. DeTar, Ziwen Fu, S. Prelovsek (2006) in preparation.

[12] C. Aubin and C. Bernard, Nucl. Phys. Proc. Suppl. 129, 182 (2004), [hep-lat/0308036]. 